\title{
A combined medical/surgical appendicitis pathway decreases pediatric CT utilization, perforation, and negative appendectomy rates
}

Sidney M. Johnson ${ }^{1 *}$, Devin P. Puapong ${ }^{1}$, Charles Peebles ${ }^{1}$, Kelli Ishihara ${ }^{2}$, Kenneth Bogenberger ${ }^{2}$ and Russell K. Woo ${ }^{1}$

\begin{abstract}
Background: We sought to improve the care of pediatric patients with possible appendicitis by decreasing unnecessary CT scanning. In an early QI initiative, we systematically emphasized the superiority of Ultrasonography (US) over CT, but did not find a decrease in $C T$ utilization in practice. We therefore redoubled our initiative using a multimodal approach. We hypothesized a combined diagnostic and treatment pathway that allowed residual diagnostic uncertainty and used both surgery and antibiotic therapy for appendicitis that would decrease the need for diagnostic CT scanning.

Results: Prior to implementation of the protocol, 33\% of ER patients with appendicitis typical abdominal pain were treated for appendicitis (with surgery); after implementation, the total number treated remained unchanged 32.5\% ( $p=$ NS), but the appendectomy rate dropped from 33 (204/619) to 23\% after implementation of the pathway (96/ $419, p<0.0005$ ) with 50 patients treated with antibiotics. There was a reduction in CT scanning (pre 39\% vs. 11\%, $p$ $<0.0001$ ) while the use of US increased (pre $30 \%$ vs. 53\%, $p<0.0001$ ). The perforation rate decreased from 12 to $5 \%(p<0.002)$ and negative appendectomy decreased from 13 to $4 \%(p<0.0001)$. Of the 50 patients treated with antibiotics, 10 eventually crossed over to surgery.

Conclusion: The use of a diagnostic and therapeutic pathway that offers antibiotic therapy for early probable appendicitis decreases the need for diagnostic CT scanning without increasing morbidity in pediatric appendicitis. Adherence to a medical/surgical treatment protocol that reserves surgery for clinically advanced appendicitis results in a reduction in CT scanning, perforation rates, negative appendectomy rates, and overall surgery for appendicitis.
\end{abstract}

\section{Background}

The use of the abdominal computed tomography (CT) scan to diagnose or confirm appendicitis is a common component of the emergency room workup for pediatric appendicitis. The availability and speed of the test, combined with its sensitivity and specificity, makes it appealing as a diagnostic tool in appendicitis $[1,2]$. That said,

\footnotetext{
* Correspondence: sidney.johnson@kapiolani.org

'John A Burns School of Medicine, University of Hawaii, Honolulu, Hawaii, USA

Full list of author information is available at the end of the article
}

widespread CT use in the evaluation of pediatric appendicitis has been criticized due to radiation exposure because the population is at higher risk of CT-related radiation injury [3, 4].

The best imaging alternative to $\mathrm{CT}$ for evaluating appendicitis is ultrasound (US) [5-7]. At our institution, an early QI (quality improvement) initiative was directed towards educating physicians to use US in favor of CT scanning. This early effort proved to be largely unsuccessful.

While our efforts in that early intervention did result in more US being done, it did not seem to decrease the

\section{Springer Open}

(- The Author(s). 2020 Open Access This article is licensed under a Creative Commons Attribution 4.0 International License, which permits use, sharing, adaptation, distribution and reproduction in any medium or format, as long as you give appropriate credit to the original author(s) and the source, provide a link to the Creative Commons licence, and indicate if changes were made. The images or other third party material in this article are included in the article's Creative Commons licence, unless indicated otherwise in a credit line to the material. If material is not included in the article's Creative Commons licence and your intended use is not permitted by statutory regulation or exceeds the permitted use, you will need to obtain permission directly from the copyright holder. To view a copy of this licence, visit http://creativecommons.org/licenses/by/4.0/. 
rate of CT scanning. When we delved into the reasons for this, it appeared that both ER physicians and surgeons were unwilling to operate on patients with early clinical symptoms or "soft positive" findings on US. This left ER physicians in a difficult position because they were unwilling to send such indeterminate patients home. Thus, both surgeons and ER physicians were uncomfortable with the remaining diagnostic uncertainty. As such, it seemed many indeterminate patients were evaluated with CT scanning in spite of the recommended US. Once we understood this, we initiated a broader institutional QI program to better address the institutional preferences that favored widespread CT scanning and then provide a functional alternative. In this effort, rather than simply recommending the preferred diagnostic modality (US), we sought to better understand and address the systematic reasons ER physicians and surgeons continued to order CTs.

After we approached the problem from this larger multidisciplinary QI process, we then sought to establish a pathway for a safe and definitive disposition from the ER, be it at home, admission, or surgery. Upon review of recent literature, we concluded that antibiotic therapy provided a safe intervention for patients with probable early appendicitis even if there was some clinical diagnostic uncertainty [8-11]. As such, we designed the pathway to allow for antibiotic therapy in probable early appendicitis as a reasonable treatment/disposition alternative for early intervention in lieu of CT scanning while reserving surgery for clinically obvious appendicitis [12-15].

\section{Methods}

We first attempted to understand why the evaluation of pediatric appendicitis favored CT scanning over US in our hospital system. We held focus meetings between surgeons, ER physicians, and radiologists to understand practice preferences.

In order to understand and address the problem, we used a QI instrument known as the Ishikawa Diagram. The Ishikawa Diagram is a quality improvement tool used to simplify and clarify systemic preferences that might lead to specific practice patterns and outcomes. The diagram helps us understand the practical reasons why physicians preferred CT scanning over US (Fig. 1).

We then crated a clinical tool to allow practitioners to follow a diagnostic and therapeutic pathway rather than CT scanning. We started with an established diagnostic scoring system (the Pediatric Appendicitis Score) to screen for probable appendicitis [16-19]. We coupled this with the US/imaging results.

To make it practically applicable, we overlaid what we understood to be best practices in medical therapy and surgery onto the PAS scoring system. This combined diagnostic and therapeutic pathway became the QI intervention. The pathway was agreed upon by consensus agreement among surgeons after review of recent randomized trials using antibiotic therapy as a treatment alternative for early appendicitis. The pathway tool appeared to give options for clinicians to provide patients with safe treatment alternatives that avoided surgery yet also allowed for treatment and disposition in the face of mild diagnostic uncertainty.

The presented algorithm, while novel, is based on established/validated diagnostic tools (the PAS score and US). While the pathway allows for the antibiotic treatment of appendicitis based on the PAS/US findings rather than reserving antibiotic therapy for strict CT criteria; it is still much more rigorous than a decision for surgery based on clinical impression or laboratory testing alone (such as would be done for suspected peritonitis). In other words, in a worst case scenario, the pathway favors unnecessary antibiotic therapy over a negative appendectomy.

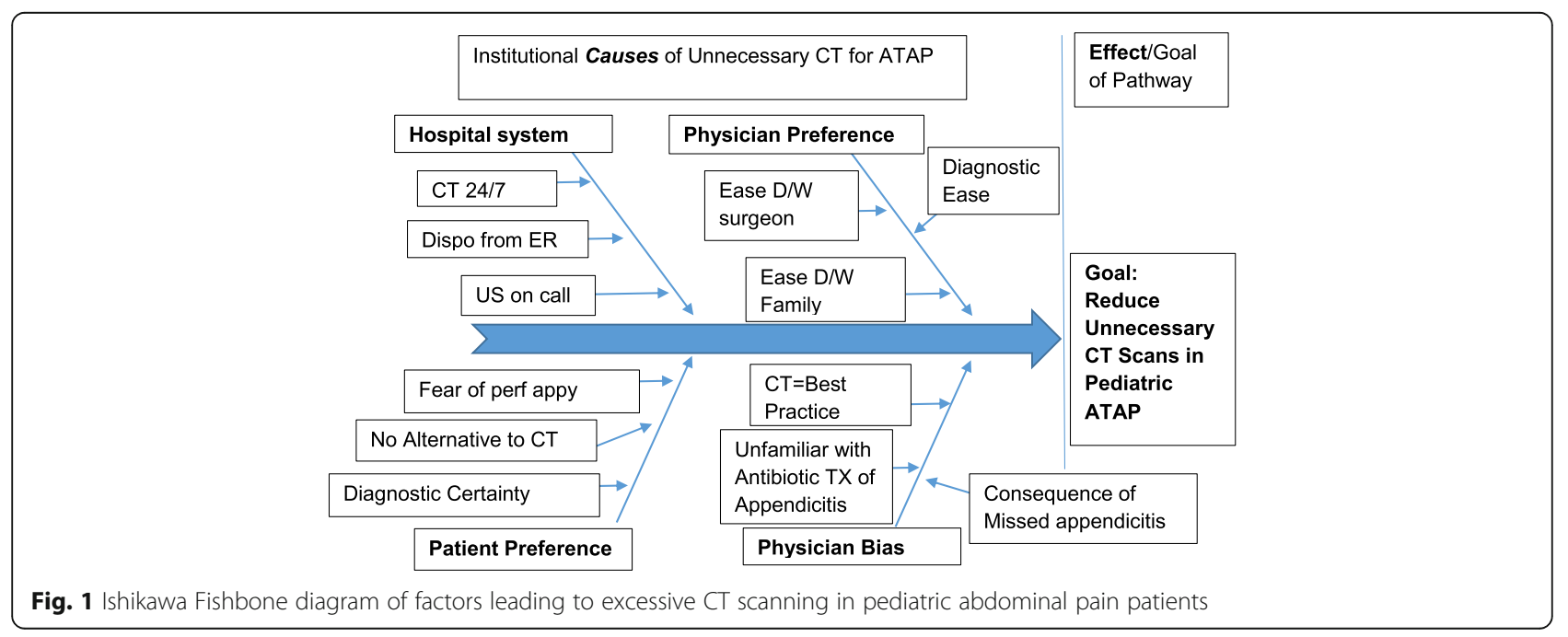


This novel approach is supported by a few assumptions that are generally accepted in appendicitis care (even if not universally practiced in all systems/institutions). Firstly, antibiotic therapy for appendicitis, when effective, appears to work best in appendicitis, when the diameter is $<10 \mathrm{~mm}$ and when therapy is implemented early. Secondly, surgeons are quite comfortable and even trained to operate on appendicitis based on clinical indications (e.g., peritonitis) in advanced, clinically obvious, and even complicated cases of appendicitis. In other words, the clinical imperatives generally supersede the diagnostic tools when it comes to the sickest patients. Thirdly, we assumed that 5 days of antibiotic therapy, even if given inappropriately, was a lesser harm than unnecessary surgery (our institutional rate of negative appendectomy was historically high, even with liberal CT scanning). Fourth, and perhaps most importantly, if antibiotic therapy can be used for both early and complicated appendicitis, and if antibiotic therapy is effectively comparable to surgery in randomized trials, there seemed to be little harm in implementing antibiotic therapy early (based on other validated clinical pathways and studies using antibiotic therapy as an alternative to surgery). At its best, the pathway allowed for equivalent therapy relative to a clinical staging system (surgery vs antibiotics for early appendicitis).

When the pathway was presented to the IRB/QI process for review, emphasis was on ensuring that all patients received an acceptable/appropriate diagnostic investigation relative to their symptoms and objective findings. At the very least, it was noted that none of the pathway outcomes were outside of general standards of care for appendicitis. It was thus determined that the systematic implementation of US with the new emphasis on the PAS clinical score was consistent with other published practices. Because the pathway thereafter employed equivalent established therapeutic options (early antibiotic therapy vs surgery), the IRB allowed the algorithm to be used as a QI tool.

The pathway was implemented in the emergency department (ER) in all patients $<18$ years of age presenting with appendicitis typical abdominal pain (ATAP), e.g., right lower quadrant pain, periumbilical pain associated with fever or vomiting, lower abdominal pain and fever, and peritonitis. The Pediatric Appendicitis Score (PAS) was paired with imaging results and medical/surgical therapy to determine patient disposition. Children were to be discharged home (low PAS), treated with IV/oral antibiotic therapy for probable early appendicitis (low PAS, early positive/suspicious US), admitted for non-operative management (low/mid PAS with appendix $<1 \mathrm{~cm}$ diameter), or taken directly to the operating room for surgical intervention (high PAS, US positive, perforation or $>1 \mathrm{~cm}$ appendix) based on PAS and imaging results. The pathway encouraged ER providers to document a clinical PAS score, obtain laboratory studies, and an US to evaluate for appendicitis in all patients with appendicitis type abdominal pain. Exceptions to US were allowed in the pathway if a CT or positive US had previously been obtained at an outside facility and in cases of clinical peritonitis with a PAS in the 9-10 range wherein the surgeon determined to operate without imaging.

Patients who received non-operative management and who showed clinical improvement in $24 \mathrm{~h}$ were discharged home with a 7-day course of antibiotics (augmentin, alternative: cipro/flagyl). Patients who demonstrated no improvement or clinical deterioration (persistent abdominal pain, worsening leukocytosis) with non-operative management underwent laparoscopic appendectomy.

The pathway was prospectively implemented for over a year, and then, outcomes were retrospectively compared to two prior years, one wherein the institution made a systematic effort to prioritize US over CT use in the evaluation of pediatric abdominal pain. It was also compared to a year when there was no institutional diagnostic pathway, imaging "best practice" initiatives, or systematic efforts in pediatric appendicitis (Fig. 2).

Patients consented for medical or surgical therapy via a standard informed consent process. All patients under 18 years of age presenting with ATAP were included in the review. Charts were reviewed from all patients who underwent appendectomy and those who had diagnostic codes consistent with ATAP in order to evaluate how CT/US and the diagnostic pathway were used. In order to conduct the review, ICD-10 codes were used to identify patients (i.e., ICD10 codes for right lower quadrant pain, periumbilical pain, lower abdominal pain, peritonitis, diffuse abdominal pain, appendicitis, perforated appendicitis, and other appendicitis).

Diagnostic interventions were also reviewed and compared between years including CBC, CRP, US, and CT scan results. In addition to diagnostic interventions, other outcome measures included crossover to surgery, negative appendectomy (pathology assessment), and rates of perforation (if deemed perforated by either surgeon or pathologist) and failure of medical management. Statistical analysis between groups was done with Fisher's exact test in consultation with institutional statisticians.

\section{Results}

The total number of patients seen for appendicitis type abdominal pain (ATAP) did not vary significantly between years and referral rates from outside hospitals that remained unchanged. After implementation of the pathway, there was no significant change in the number of patients evaluated (mean $=346 /$ year).

Prior to implementation of the protocol, 33\% of ER patients with appendicitis typical abdominal pain were treated with appendectomy. The rate of appendectomy for appendicitis typical abdominal pain did not vary between the two retrospective years. During prior years, 


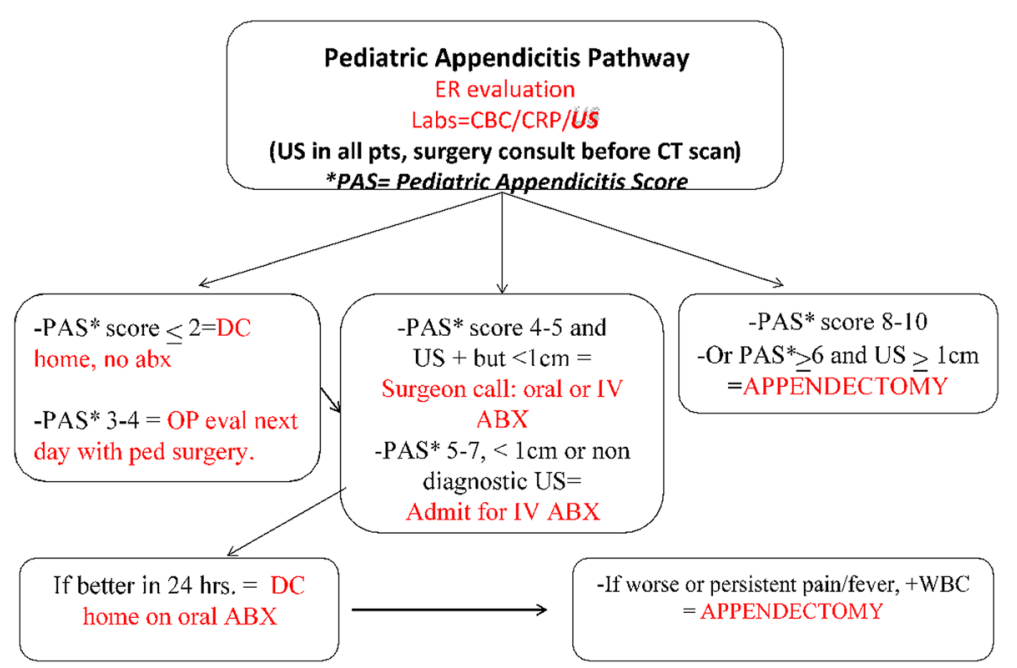

Fig. 2 Pediatric appendicitis pathway implemented in our study

appendectomy rate on patients with appendicitis typical abdominal pain was 33\% (204/619-2 years).

During the year of pathway utilization, the number of patients treated for appendicitis did not change significantly $(32.5 \%(p=\mathrm{NS})$ were treated for appendicitis). However, the way appendicitis was treated varied as medical therapy for early appendicitis was integrated into systemic care. After implementation of the pathway, the overall rate of appendectomy dropped to $23 \%$ (96/419, $p<0.0005)$. Fifty patients were treated with antibiotics. Of the 50 patients treated with antibiotics for appendicitis, 10 crossed over to surgical therapy without undue morbidity. Rates of perforation in the early antibiotic group were significantly lower than the surgical group.

Use of the protocol resulted in a reduction in CT scanning (pre $39 \%$ vs. post $11 \%, p<0.0001$ ) and also increased the use of US over CT (pre $30 \%$ vs. $53 \%, p<0.0001$ ) (Fig. 3). It is important to keep in mind that these are rates for all ER patients with ATAP, and many were clinically deemed to not meet the criteria for either CT or US. While CT scanning rates improved significantly with the pathway, it was not avoided completely. We found that this was for two main reasons. Most commonly it was because patients were referred from outside centers with CT scanning done prior to referral. There were also select patients who underwent CT scanning at our facility at the discretion of ER and surgical providers due to individual circumstances (CT was still offered to patients as a diagnostic alternative to treatment with antibiotic therapy). Moreover, based on US findings, CT scanning was occasionally necessary for appropriate treatment (e.g., for percutaneous drainage of an advanced peri-appendiceal abscess seen on US).

Overall, the pathway was largely successful at our institution, and the protocol continues to be utilized. After the implantation of the pathway, the vast majority of CTs were done at referral centers (Fig. 4). Moreover,

CT use for ATAP before/after protocol

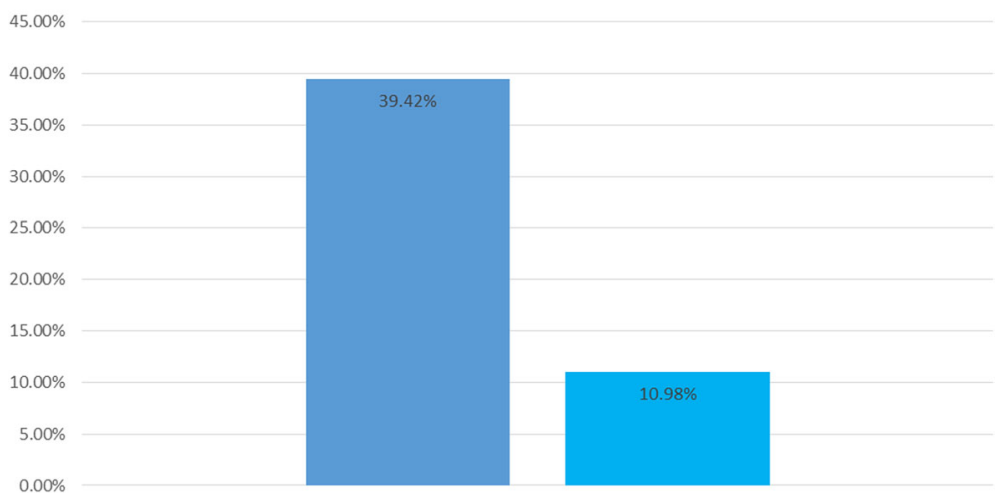

Fig. 3 CT utilization at our institution before/after protocol was implemented 


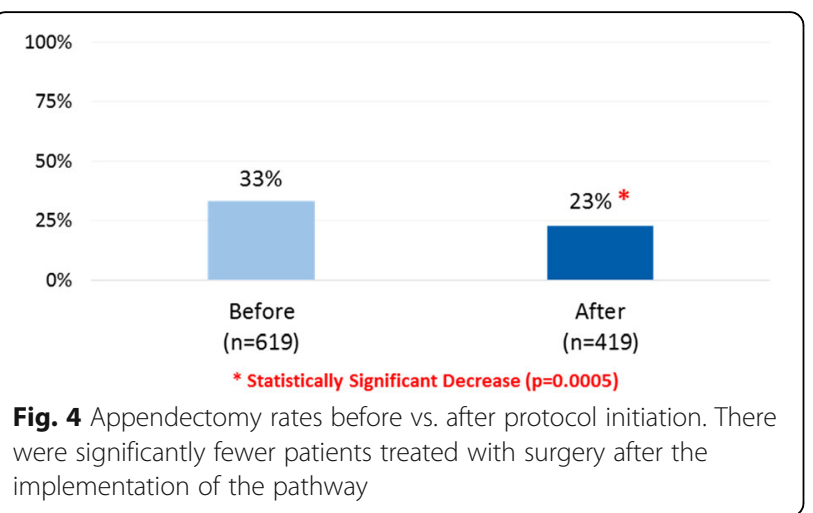

once the pathway was implemented and antibiotic treatment was integrated as an early therapeutic option, the perforation rate decreased from 12 to $5 \%(p<0.002)$ and negative appendectomy decreased from 13 to $4 \%$ ( $p<$ 0.0001). Of the 50 patients treated with antibiotics, 10 eventually crossed over to surgery (Figs. 4 and 6).

\section{Discussion}

The goal of this quality improvement effort was to reduce unnecessary CT scanning for the evaluation of abdominal pain [20]. The early best practice initiatives using US were generally ineffective within our system. Prior to implementation of the pathway, even with systemic efforts improved at US utilization, the overall CT use was not significantly affected (Fig. 5).

Because of this, a new multidisciplinary QI review was undertaken in order to establish the pathway. The review revealed that physicians preferred the definitive diagnostic certainty provided by CT. CT scanning eased difficult conversations with parents when the treatment disposition was in question (surgery, admit, or send home). In other words, practitioners felt the CT was the "gold standard," especially when US was equivocal or indeterminate. Practitioners did not feel that US provided definitive disposition options when families asked if there was a better available test. This was compounded by the fact that the radiologists often recommended "further imaging with CT based on clinical correlation." As such, ER physicians and surgeons alike felt obligated to obtain diagnostic accuracy in order to best inform families.

We therefore changed tactics and used established quality improvement tools to look at our systematic practice. With the use of these tools, it became clear that institutional preferences for CT were deeply embedded in physician practice. The main problems clarified by QI tools were that US did not appear to be sufficiently definitive, was not as readily available as CT, and left caregivers with diagnostic uncertainty. This left ER physician and surgeons without a clear disposition or treatment intervention, and physicians were not comfortable with admitting/observing when a more definitive diagnostic option was available (CT). We speculated this practice was likely reflected provider indecision or difficulty with disposition for abdominal pain patients with indeterminate or early appendicitis based on US alone. Those practice biases explained why providers continued to rely on CT to provide immediate disposition and treatment options for patients and surgical and ER caregivers.

Interestingly, our results indicate that the broad use of CT was not improving the clinical practice in the way physicians believed it was. Broad use of CT did not improve our ability to decrease perforation and negative appendectomy rates prior to implementation of the pathway. Moreover, given our moderately high preintervention negative appendectomy rate, it appears (again in retrospect) that liberal CT use may have actually increased our appendectomy rate and thereby increased the rate of unnecessary surgery [21] (Figs. 5 and 6). Other centers and studies have also indicated a

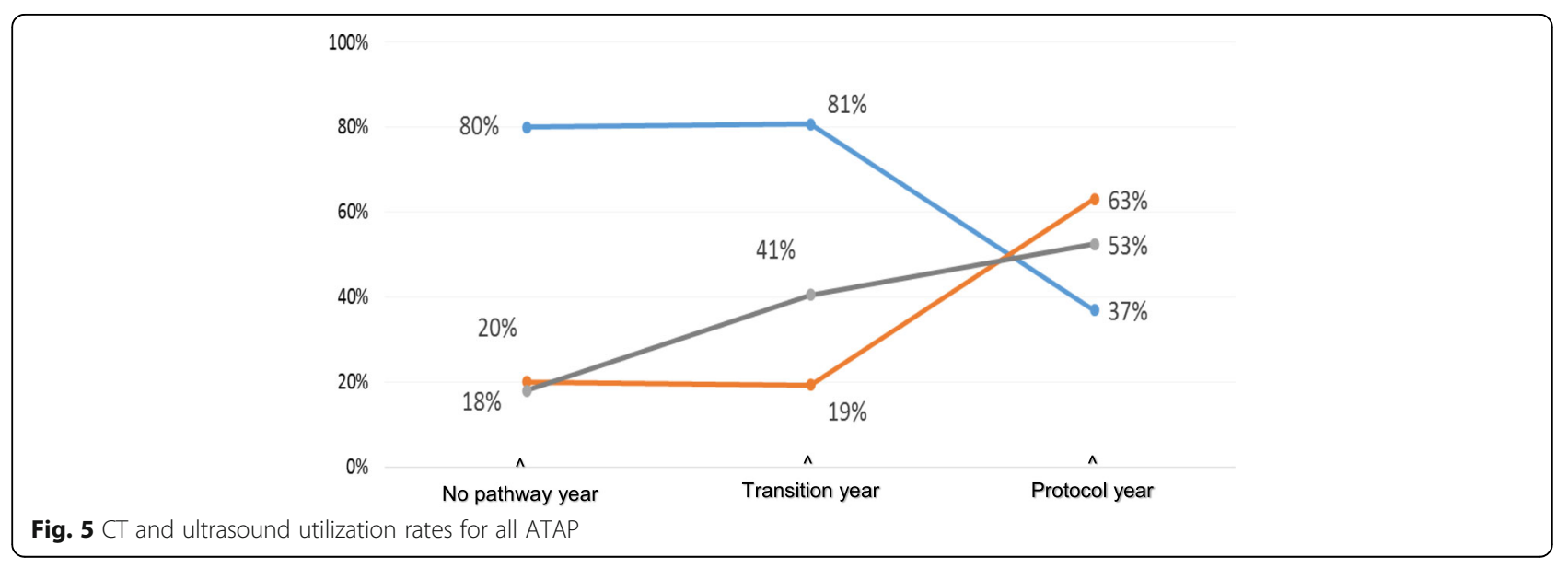




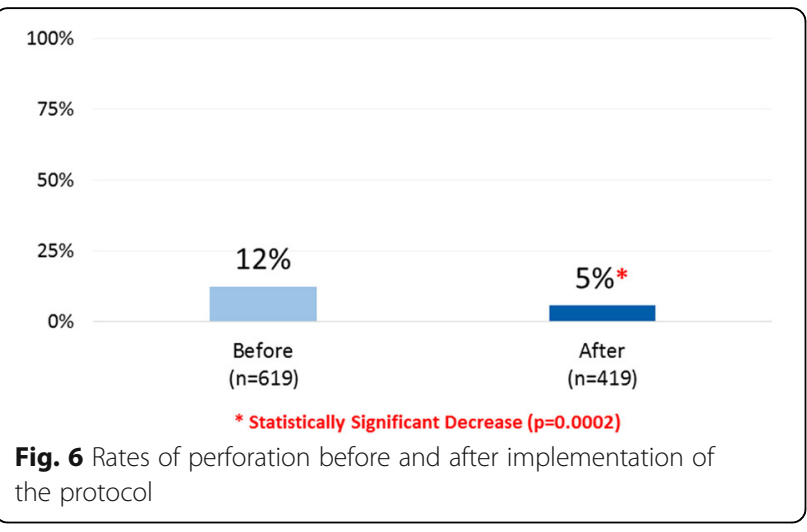

paradoxical increased rate of appendectomy with liberal CT utilization [22].

After the identification of systematic problems favoring CT utilization, we identified antibiotic therapy as a pragmatic solution to both the diagnostic uncertainty and disposition problems noted by caregivers. As we developed our appendicitis pathway, we realized that the key intervention was not to simply encourage or mandate the use of US, but to provide a definitive disposition to supplant the underlying reason physicians were using CT [23]. We reasoned that if the current surgical evidence supported antibiotic therapy for early appendicitis as equivalent to surgery, there could be little harm in making a diagnostic and therapeutic pathway that prioritized a clinical diagnosis of appendicitis and gave disposition options that were at least as safe and definitive as $\mathrm{CT}$ and surgery alone.

We believed there were two potential problems with the pathway. Firstly, we took an established scoring system (PAS) and added antibiotic therapy to it which added a layer of complexity for caregivers. Such was also not the original design or intent of the PAS, and we did not know how well the pathway would work practically with the added complexity. That said, upon review with the care teams, the complexity appeared understandable and manageable. It also appeared safe based upon reviews of recent literature about antibiotic therapy for pediatric appendicitis $[8,24,25]$.

The pathway also raised a second potential problem: possible overtreatment with antibiotics. After we assessed the total number of patients that presented with appendicitis typical abdominal pain (ATAP) that fit the criteria using the PAS score and the US criteria, we decided that the total number of patients who might be overtreated with antibiotics would be relatively low. In the end, we determined this would need to be one of the outcome metrics of the quality improvement initiative.

That noted, our results indicate that by adhering to the pathway, we did not grossly overtreat with antibiotics. The total number of patients treated for ATAP did not differ significantly between years, nor did the total number of appendicitis patients differ between years (including those treated with antibiotics). So while it is likely some were treated earlier and more liberally for appendicitis, it does not appear that the number was high. Moreover, the result seems to be a favorable trade off in that the total number of patients treated with surgery decreased significantly. Moreover, the rate of negative appendectomy also dropped indicating that, if patients were overtreated with antibiotics, the trade-off was fewer unnecessary surgeries.

This review demonstrates that implementation of a structured pathway or protocol in assessing pediatric patients with appendicitis type abdominal pain has several advantages. The first is that it did decrease the number of unnecessary CT scans in our population. The second advantage of the pathway is it leads to a reduction in the amount of negative appendectomies or, in other words, unnecessary surgeries, without increasing the risk of complicated or perforated appendicitis. In addition, overall surgery rates, negative appendectomy rates, and appendiceal perforation rates all decreased significantly when compared to management prior to implementation of the protocol. Overall, the institutional experience has been favorable, and the pathway continues to be utilized. Our experience is that many families wish to try antibiotic therapy as a comparable alterative to surgery in early appendicitis.

There are limitations to the study in that it was conducted at a single institution. Implementing the pathway in other facilities would strengthen the conclusions. The pathway also has inherent limitations as a quality improvement project as the results may be dependent on improving weaknesses in a local/contained medical system. Additionally, though we observed improved clinical outcomes, many of these were observed secondarily and could be related to measurement effect/pathway implantation. We also experienced treatment failures with some patients crossing over to surgery after failing medical management (20\%). These failure results were consistent with the results of pediatric RCTs comparing medical therapy to surgery for appendicitis. That said, this also correlated with a decreased rate of perforation and complicated appendicitis supporting the notion that early medical therapy is a safe alternative.

Again, it is worth noting that in one of the comparison years, we did make a prior institutional effort to improve our accuracy with US. Unfortunately, our efforts simply resulted in more US being done without a decrease in CT scanning. This speaks to the importance of understanding that QI processes are not single-pronged (e.g., not as simple as telling physicians to use US). Specifically, our experience highlights how QI processes and problems are multifaceted and the results of the interventions can also reach beyond the intended outcome. 
In our case, it seemed ER physicians and surgeons were unwilling to operate on patients with early clinical symptoms or "soft" findings on US, and as such, most indeterminate patients were further evaluated with CT scanning in spite of the US pathway. The problem then was that patients who then also had "soft" findings of appendicitis (CT positive without clear surgical indications such as point tenderness or peritonitis) were then taken for surgery because the surgeons felt obligated to adhere to the CT findings. This behavior likely accounts for the $14 \%$ rate of negative appendectomy when US and CT were used without the pathway.

This issue highlights the strength of the pathway in that it offers a simple, low-risk, and surgical alternative for early or indeterminate appendicitis: antibiotic therapy. Although the goal of the QI intervention was to decrease unnecessary CT scanning by providing viable diagnostic and therapeutic options, it turns out that the probable value of the pathway was that it allowed surgeons and ER physicians alike to approach early appendicitis differently, thus decreasing not only the rate of CT scanning, but also the rate of perforation and negative appendectomy. Instead of worrying about indeterminate diagnostic results (the reason CTs were being over-ordered), a safe therapeutic option was implemented early (antibiotic therapy) in the patient's disease course. In lieu of "observation," serial abdominal exams or repeated visits to the ER or PCP, the pathway implements early antibiotic therapy. This was an unforeseen and unanticipated outcome of the QI pathway.

\section{Conclusion}

The use of a diagnostic and therapeutic pathway that offers antibiotic therapy for early probable appendicitis decreases the need for diagnostic CT scanning without increasing morbidity in pediatric appendicitis. Adherence to a med$\mathrm{ical} /$ surgical treatment protocol that reserves surgery for clinically advanced appendicitis results in a reduction in CT scanning, perforation rates, negative appendectomy rates, and overall surgery for appendicitis.

\section{Abbreviations}

QI: Quality improvement; ER: Emergency room; CRP: C-reactive protein; US: Ultrasonography; CBC: Complete blood count; ATAP: Appendicitis typical abdominal pain; PAS: Pediatric Appendicitis Score; CT: Computed tomography scan; RCT: Randomized controlled trial

\section{Acknowledgements}

The Hawaii Pacific Health Research Institute.

\section{Authors' contributions}

SMJ was responsible for the conception, data gathering/analysis, manuscript writing. $\mathrm{CP}$ and $\mathrm{KI}$ were responsible for the manuscript writing/revision. KB was responsible for manuscript revisions. DPP and RKW were responsible for the conception, analysis, and manuscript revision. All authors have read and approved the manuscript.

\section{Funding}

none.
Availability of data and materials

Available upon request.

\section{Ethics approval and consent to participate}

The review of this appendicitis pathway was approved by the Hawaii Pacific Heath Research Institute as a quality improvement project under their Training in Health Care Improvement Program. The need for informed consent was waived as the protocol was instituted as a quality improvement initiative and the data was collected retrospectively. Approval IRB waiver was obtained from the Hawaii Pacific Health Research Institute. HPHRI 2016-041.

\section{Consent for publication}

The authors give consent for publication.

\section{Competing interests}

The authors declare that they have no competing interests.

\section{Author details}

'John A Burns School of Medicine, University of Hawaii, Honolulu, Hawaii, USA. ${ }^{2}$ Tripler Army Medical Center, Honolulu, Hawaii, USA.

Received: 14 October 2019 Accepted: 2 March 2020

Published online: 30 March 2020

\section{References}

1. McGory ML, Zingmond DS, Nanayakkara D, Maggard MA, Ko CY. Negative appendectomy rate: influence of CT scans. Am Surg. 2005;71(10):803-8.

2. Frei SP, Bond WF, Bazuro RK, Richardson DM, Sierzega GM, Reed JF. Appendicitis outcomes with increasing computed tomographic scanning. Am J Emerg Med. 2008:26(1):39-44.

3. Brenner DJ, Elliston CD, Hall EJ, Berdon WE. Estimated risks of radiation. Ajr. 2001;176(2):289-96.

4. Brenner DJ, Hall EJ. Computed tomography--an increasing source of radiation exposure. N Engl J Med. 2007;357(22):2277-84.

5. Polites SF, Mohamed Ml, Habermann EB, et al. A simple algorithm reduces computed tomography use in the diagnosis of appendicitis in children. Surgery. 2014

6. Bachur RG, Levy JA, Callahan MJ, Rangel SJ, Monuteaux MC. Effect of reduction in the use of computed tomography on clinical outcomes of appendicitis. JAMA Pediatr. 2015.

7. Kotagal M, Richards MK, Chapman T, et al. Improving ultrasound quality to reduce computed tomography use in pediatric appendicitis: the Safe and Sound campaign. Am J Surgery. 2015;209(5):896-900. https://doi.org/10. 1016/j.amjsurg.2014.12.029.

8. Xu Y, Jeffrey RB, Chang ST, DiMaio MA, Olcott EW. Sonographic differentiation of complicated from uncomplicated appendicitis: implications for antibiotics-first therapy. J Ultrasound Med. 2016;31..

9. Eriksson S, Granström L. Randomized controlled trial of appendicectomy versus antibiotic therapy for acute appendicitis. Br J Surg. 1995;82(2):166-9.

10. Salminen $\mathrm{P}$, Paajanen $\mathrm{H}$, Rautio $\mathrm{T}$, et al. Antibiotic therapy vs appendectomy for treatment of uncomplicated acute appendicitis: The APPAC randomized clinical trial. JAMA. 2015;313(23):2340-8.

11. Gonzalez DO, Deans KJ, Minneci PC. Role of non-operative management in pediatric appendicitis. Semin Pediatr Surg. 2016;25(4):204-7.

12. Talan DA, Saltzman DJ, Mower WR, et al. For the Olive View-UCLA Appendicitis Study Group. Antibiotics-first versus surgery for appendicitis: a US pilot randomized controlled trial allowing outpatient antibiotic management. Ann Emerg Med. 2016.

13. Hansson J, Körner U, Khorram-Manesh A, Solberg A, Lundholm K. Randomized clinical trial of antibiotic therapy versus appendicectomy as primary treatment of acute appendicitis in unselected patients. Br J Surg. 2009;96(5):473-81.

14. Styrud J, Eriksson S, Nilsson I, et al. Appendectomy versus antibiotic treatment in acute appendicitis: a prospective multicenter randomized controlled trial. World J Surg. 2006;30(6):1033-7.

15. Vons C, Barry C, Maitre $S$, et al. Amoxicillin plus clavulanic acid versus appendicectomy for treatment of acute uncomplicated appendicitis: an open-label, non-inferiority, randomised controlled trial. Lancet. 2011; 377(9777):1573-9.

16. Alvarado A. A practical score for the early diagnosis of acute appendicitis. Ann Emerg Med. 1986;15(5):557-64. 
17. Schneider C, Kharbanda A, Bachur R. Evaluating appendicitis scoring systems using a prospective pediatric cohort. Ann Emerg Med. 2007;49(6): 778-84.

18. Gronroos JM. Do normal leucocyte count and C-reactive protein value exclude acute appendicitis in children? Acta Paediatr. 2001;90(6):649-51.

19. Gronroos JM, Gronroos P. Leucocyte count and C-reactive protein in the diagnosis of acute appendicitis. Br J Surg. 1999;86(4):501-4.

20. Fair BA, Kubasiak JC, SF JP, Mohamed MI, Habermann EB, et al. A simple algorithm reduces computed tomography use in the diagnosis of appendicitis in children. Surgery. 2014.

21. Martin $A E$, et al. CT scans may not reduce the negative appendectomy rate in children. J Pediatric Surgery. 2004;39(6):886-90.

22. Livingston EA, Woodward WA, Sarosi GA, Haley RW. Disconnect between incidence of non-perforated and perforated appendicitis. Ann Surg. 2007; 245(6):886-92.

23. Abou-Nukta F, Bakhos C, Arroyo K, Koo Y, Martin J, Reinhold R, et al. Effects of delaying appendectomy for acute appendicitis for 12 to 24 hours. Arch Surg. 2006. 141(5):504-506; discussion 506-7.

24. Chang Y-J, Chao H-C, Chen C-L, Chen S-Y, Yan D-C, Tsai M-H. C-reactive protein may predict the recurrence of appendicitis in children formerly with appendiceal mass after successful non-operative treatment. Pediatr Neonatol. 2016:(2016):1-5.

25. Bickell NA, Aufses AH Jr, Rojas M, Bodian C. How time affects the risk of rupture in appendicitis. J Am Coll Surg. 2006;202(3):401-6.

\section{Publisher's Note}

Springer Nature remains neutral with regard to jurisdictional claims in published maps and institutional affiliations.

\section{Submit your manuscript to a SpringerOpen ${ }^{\circ}$ journal and benefit from:}

- Convenient online submission

- Rigorous peer review

- Open access: articles freely available online

- High visibility within the field

- Retaining the copyright to your article 